\title{
Prognosefaktoren bei therapieinduzierten myeloischen Neoplasien
}

Therapieinduzierte myeloische Neoplasien (t-MN) nach früherer Strahlen- und/oder Chemotherapie beinhalten akute myeloische Leukämien und myelodysplastische Syndrome. Welche Krankheitsverläufe und Prognosen verbergen sich dahinter im Einzelnen und welchen Einfluss hat die Therapie?

U m Charakteristika, Entstehung und

Prognose der t-MN besser zu verstehen, erfasste das Italian Network on Secondary Leukemias die Daten von 277 erwachsenen t-MN-Patienten (104 retrospektiv, 173 prospektiv; 157 AML, 120 MDS). Primärdiagnose war bei 111 Patienten eine hämatologische Neoplasie (70 Hodgkin-Lymphome [HL], 18 Non-Hodgkin-Lymphome [NHL], 12 multiple Myelome [MM] und 6 chronische lymphatische Leukämien [CLL]) bei 155 ein solider Tumor (43\% davon ein Mammakarzinom) und bei 11 eine Autoimmunerkrankung. Primär waren die Patien- ten mit Strahlentherapie (RT, $\mathrm{n}=43$ ), Chemotherapie (CT, $\mathrm{n}=133$ ) oder $\mathrm{Ra}$ diochemotherapie (RCT, $\mathrm{n}=101$ ) behandelt worden. Das Intervall zwischen Primärtherapie und $\mathrm{t}-\mathrm{MN}$ betrug median 5,7 Jahre, bei einer alleinigen Strahlentherapie war es mit 11,2 Jahren signifikant länger als nach CT oder RCT (7,1 Jahre, $p=0,0005)$. Eine Kombination aus Alkylans und Topoisomerase-IIHemmer verkürzte die Latenzzeit im Vergleich zu einem Alkylans alleine von 8,4 auf 6 Jahre $(p=0,02)$.

Das Überleben aller Patienten mit t-MN betrug ab Diagnose median 14,8
Monate und wurde durch die Therapie entscheidend beeinflusst. Am längsten überlebten mit 58,8 Monaten Patienten nach allogener Stammzelltransplantation gegenüber 12,1 Monaten nach anderen Therapien $(p<0,0001)$. Entscheidenden negativen Einfluss hatte auch ein ungünstiger Karyotyp (Hazard Ratio 0,447 gegenüber günstig/intermediär; $\mathrm{p}$ $<0,0001$ ) und ein höheres Alter ( $>60$ vs. $\leq 60$ Jahre) - ähnlich wei bei der De-novo-AML - sowie eine Anämie.

Fazit: Die Therapiestrategie sollte bei jüngeren Patienten mit t-MN nicht auf die klassische CT verzichten und auch eine allogene Stammzelltransplantation in Erwägung ziehen. Brigitte Schalhorn

Fianchi L et al. Characteristics and outcome of therapy-related myeloid neoplasms: report from the Italian Network on Secondary Leukemias. Am J Hematol. 2015 May;90(5):E80-5.

\section{CD8-Zellen im Transplantat entscheiden}

\section{Nach allogener hämatopoetischer Stammzelltransplantation mit einer Konditionierung von reduzierter Intensität (alloRIC) ist die Rezidivwahrschein- lichkeit hoch. Spielt der Gehalt an T-Zellen im Transplantat eine Rolle?}

Re ezidive treten bei $25-60 \%$ der Patienten auf, die wegen einer hämatologischen Neoplasie mit einer alloRIC behandelt werden. In einer aktuellen Studie wurden mögliche Assoziationen zwischen T-Zell-Konzentrationen im Transplantat $\left(\mathrm{CD} 8^{\text {hi }}=\right.$ hohe und $\mathrm{CD} 8^{\text {lo }}=$ niedrige Konzentration) und der Prognose bei 200 Patienten untersucht, die sich einer alloRIC unterzogen hatten. Außerdem wurden 21 Stammzellspender hinsichtlich klinischer und immunologischer Faktoren genauer unter die Lupe genommen. Das mediane Followup betrug 29,4 Monate.

Höhere CD8-Zelldosen waren mit einem niedrigeren Risiko für ein Rezidiv (adjustierte Hazard Ratio [aHR] 0,43; p $=0,009$ ), einem verbesserten rezidivfreien Überleben (aHR 0,50; $\mathrm{p}=0,006)$ und einem verbesserten Gesamtüberleben (aHR 0,57; $\mathrm{p}=0,04)$ verbunden. Dies galt für Patienten mit allen Risikostufen der Erkrankung. So hatten beispielsweise Patienten mit Hochrisiko-Krankheit, die ein $\mathrm{CD}^{\text {lo }}$-Transplantat erhalten hatten, mit einer Gesamtüberlebensrate von nur 26,2\% im 1. Jahr nach der Transplantation eine ungünstige Prognose. Patienten mit Hochrisiko-Krankheit und CD8 ${ }^{\text {hi }}$-Transplantat hingegen hatten eine ähnliche Prognose wie die Patienten mit Niedrigrisiko-Krankheit und CD8 ${ }^{\text {lo }}$-Transplantat. Dabei kam es weder zu einer signifikanten Häufung der Graft-versus-Host-Krankheit, noch erhöhte sich die nicht rezidivbedingte Mortalität. Die Schwelle zwischen CD $8^{\text {hi }}$ und CD8 ${ }^{\text {lo }}$ Transplantaten im Hinblick auf die Überlebenswahrscheinlichkeit lag bei $0,72 \times 10^{8} \mathrm{CD} 8-Z$-Zllen/kg Körpergewicht $(\mathrm{p}=0,007)$.

Das Alter des Spenders korrelierte invers mit der CD8-Dosis im Transplantat.
Bei älteren Spendern kamen $\mathrm{CD} 8^{\text {hi }}$ Transplantate kaum vor, hingegen bei $53 \%$ der Spender unter 30 Jahren. Verglichen mit Empfängern von Spenden älterer Geschwister (durchweg CD8 ${ }^{\text {lo }}$ Transplantate) hatten Empfänger von CD8 ${ }^{\text {hi }}$-Transplantaten jüngerer NichtVerwandter ein signifikant besseres Überleben $(\mathrm{p}=0,03)$. Die Senkung des Rezidivrisikos und die Verbesserung des Überlebens bei hohen CD8-Zelldosen sind vermutlich mit schnellerem Anwachsen der Spender-T-Zellen assoziiert.

Fazit: Höhere CD8-Werte im Transplantat - nahezu ausschließlich bei jungen Spendern - sind bei alloRIC mit einem besseren Überleben verbunden. Da die alloRIC gerade bei älteren Patienten, die oft keine myeloablative Konditionierung vertragen, die einzige kurative Option ist, ist die Optimierung des Transplantats entscheidend.

Kathrin von Kieseritzky

Reshef R. et al. High Graft CD8 Cell Dose Predicts Improved Survival and Enables Better Donor Selection in Allogeneic Stem-Cell Transplantation With Reduced-Intensity Conditioning. J Clin Oncol. 2015;33(21):2392-8. 